\title{
A REVIEW ON IOT BASED TRAFFIC MANAGEMENT SYSTEM
}

\author{
Lavanya N R \\ Student, Department of ECE \\ Vidhyavardhaka College of engineering, \\ Mysusru, karnataka. India.
}

\begin{abstract}
Traffic jams are prominent in almost every urban area across the world. Traffic management is one of the crucial thing where monitoring and controlling of traffic needs to be done with real time information. Considering the capability of IoT to solve many existing issues around us, in this paper we have discussed IoT based traffic control systems with its hardware and software arrangements and how it performs in a view of a city or road with its merits, demerits and uses. From the above study we are able to interpret that an IoT based traffic control system has its own boon and banes and can be specially made and used duly.
\end{abstract}

Keywords- IoT, Image processing, WSN, Node-Red, ultrasonic sensor

\section{INTRODUCTION}

Traffic jam is a great trouble in the world today as it affects life regime of urban areas and also perturbs the environment around us. The vehicle density on roads has seen a huge hike because of growth in population and expansion of cities. This makes the traffic crowding a main concern of many big cities around the world. Traffic jams have negative effects on the fecundity, fierceness and economy of the country. Hence researchers have come up with numerous solutions to this issue.

The research says that in the year 2014, 54\% of world's total population resided in urban areas. The following years also have had the increase in urban population which has put constraint on transport system. As the cost of living in business areas is high the employees living far from work place have to travel from their place of living. Though the making of roads bigger seems to be sudden response to the issue, cities must aim on running their roads smarter.

The growth and improvement in internet with regard to bandwidth and speed, internet of things has

been able to take the market on different node and has made way for numerous inventions. IoT is enabled due to the contribution of fields like embedded systems, automation, wireless sensor network and control systems. Smart traffic control is possible by the interaction amongst the elements of transport system. And all the feature of transport systems has the employment of IoT. Platform of IoT facilitates continuous inspection of traffic through wireless sensors and it ensures to alert when exceptions in management happen.

\author{
Panchami S V \\ Assistant Professor, Department of ECE \\ Vidhyavardhaka College of engineering, \\ Mysusru, karnataka, India.
}

The combination of traffic systems with the IoT will assure the smart security along with the road safety. The building of embedded system by combining of many sensors with the concerned website which enables the access to real time information will result in the more effective and safe traffic control.

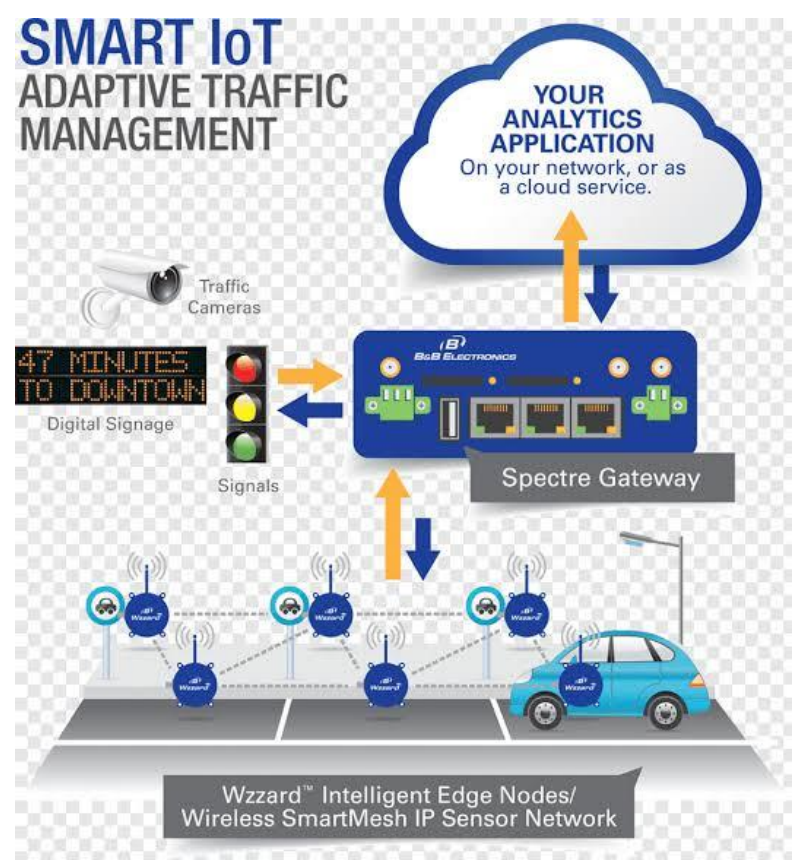

Fig 1: Architecture of IoT based traffic control system

As shown in the figure 1 traffic cameras are installed which capture the density of the vehicles on the road. The sensors in the road also collect information of the traffic. This data collected is given to the server. It has used spectra advanced wireless gateway which allows smooth transfer of data to cloud service or server. Based on the collected data on density of vehicles on road the traffic signal time is allotted.

Therefore, devising of system for traffic control, using IoT by automated vehicle counting and controlling traffic lights is crucial for smart and safe roads. 


\section{International Journal of Engineering Applied Sciences and Technology, 2020 \\ Vol. 5, Issue 1, ISSN No. 2455-2143, Pages 612-615 \\ Published Online May 2020 in IJEAST (http://www.ijeast.com)}

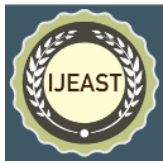

\section{RELATED WORK}

The authors Mounir BOUHEDDA, Hamza BENYEZZA, Ibrahim METALI, Sofiane TCHOKETCH KEBIR, Samia REBOUH in 2019 have discussed, a platform of IoT where a NodeRed server is advanced and an intelligent controller rooted on fuzzy logic is executed using python language to manage the road intersections. The communication between the server and the road intersection is made possible by RF communication. The fuzzy control logic, which has been successfully designed and executed using Node-Red server reveals that this system can be implemented by applying any complex algorithm without facing obstacle of the memory size of microcontroller[1].

The authors Anilloy Frank, Yseer Slim Khamis Al Aamri, Amer Zayegh in 2019 have proposed, solution to control the traffic crowding by usage of Raspberry $\mathrm{Pi}$ along with Image processing abilities. The system is advanced by using IoT for observing and controlling traffic signal based on density applying image processing. The system has principal focus on the captured image using the camera. The apprehended image would be again checked with a present image that was laden in the server to calculate the density. The traffic motion is controlled rooted on density of vehicle. This decreases the total stand by time and brings out a smooth traffic flow[2].

The authors Satbir Singh, Baldev Singh, Ramandeep Baljit Singh, Amitava Das in 2019 have proposed the system in which, the vehicles are counted before reaching the traffic jam points and information of vehicle density is sent for controlling of traffic jam. The well timed data of the traffic congestion is conveyed by internet and cloud for controlling the flow of vehicles. For real time calculation of vehicles, Open-CV is used. The system controls traffic on regional and concentrated servers by using the IOT and Intelligent Image Processing. Raspberry Pi rooted system has been used as hardware which is low cost and movable system to aid reducing the traffic crowding on roads at apex points[3].

The authors Mehal Zaman Talukder, Sheikh Shadab Towqir, Arifur Remon, Hasan U. Zaman in 2017 have discussed a system in which, microcomputer, multiple ultrasonic and Raspberry $\mathrm{Pi}$ are applied in every road to find the traffic density and lane is operated considering the vehicle density. Traffic information is continuously updated to website. This characteristic could be used for real time data of a intersection and change route duly. This can decrease the time wasted by people in traffic signal junction and decrease traffic crowding on busy roads caused due to signals on unoccupied lanes[4].

The authors Pampa Sadhukhan, Firoz Gazi in 2018 have discussed a system in which, the density of traffic at road crossing is measured by applying ultrasonic sensor node (USN) and it in turn sets the signal time considering the counted values of vehicle density. It also utilizes a emerging technique of approximating the density of traffic crowding by usage of USN. The examination of operation of this discussed traffic control system by test bed is the expected future[5].
The authors Anna Merine George, V.I.George, Mary Ann George, in the year 2018, have discussed the system in which traffic data is examined applying techniques of Image processing and Adaptive Neuro fuzzy. A camera is staged crabwise the traffic signal light to get the sequences of images of road traffic. This image is transferred to the cloud server applying platform of ThingSpeak. The control signal given by the adaptive fuzzy controller decides the order of a lane to receive the needed traffic signal light is decided by adaptive fuzzy controller by examining of traffic information. The comfort and security for the drivers are achieved by augmented reality[6].

The researchers Varsha Sahadev Nagmode, Prof.Dr.S.M.Rajbhoj, in 2017, have discussed the system that applies technology of sensing to observe information of traffic of vehicle by applying ultrasonic sensors to find traffic hierarchy and send data to unit of controller which processes information and exhibit it on server. This method is applied to decrease traffic issues and make priority for emergency vehicles at signal. If heavy traffic is found in any road, traffic signal time is increased for vehicle to pass. The embedded system which applies wireless sensor network gives a framework for observing and managing any traffic associated real-time data. This system is authentic for users [7].

The authors Rafik Zitouni, Jeremy Petit, Aghiles Djoudi and Laurent George, have proposed the system which applies designing, framework, and examining of MQTT protocol. This paper discusses a new model of urban traffic light control based on a network of IoT (IoT UTLC). The goal is to connect both lanes and armature with traffic signal light by means of IoT. They have framed this system by choosing protocol and mediums of wireless sensor network(WSN). Message queuing telemetry transport (MQTT) protocol has been combined to control QoS. This system has been analyzed for the correlation between the protocol of MQTT and low traffic crowding [8].

\section{ADVANTAGES AND DISADVANTAGES}

Many researchers have worked on devising and execution of IoT based traffic control systems. The execution of these systems has been successful. The system would be able to perform involuntarily rooted on data collection of vehicle density image received by the server from the particular region. The results acquired reveal that the platform of IoT eases the observing of traffic in the lanes. Police officers would be able to observe taffic situation in real-time. One step forward MATLAB could be connected to platform of ThingSpeak to evaluate the traffic information. Independent driving operations are made easy by this system. The researchers have put efforts to make these systems well-grounded users. any of the complex algorithms can be applied for the execution of these systems and microcontroller's memory proportions do not pose trouble. And 


\section{International Journal of Engineering Applied Sciences and Technology, 2020 \\ Vol. 5, Issue 1, ISSN No. 2455-2143, Pages 612-615 \\ Published Online May 2020 in IJEAST (http://www.ijeast.com)}

few systems are made to direct signal time, as per levels of traffic in the roads, also assures preference to exigency vehicle. They can be performed with ease and fit out at any location and these systems are less expensive.

Nevertheless, there should be execution of processes by organizations to secure sensitive information as there is increase in complexity due to numerous sensors and network interconnected devices, every IoT system has to be tractable with policies of security. This compels it speculative to control all the finishing line. A lot of IP address is required to be managed, toil with larger volumes of information, and deal with more complicated IT framework. Networks are required to be scaled in order to accommodate as the output information of IoT grows due to addition of workflows and controls. It can get better by application of encryption, authentication of the system, or by controlling user access.

\section{APPLICATIONS}

In Cities data from feeds of CCTV can be extracted and vehicle associated information can be sent to traffic control stations. This information can be integrated with information from the smart parking sensors, smart traffic signals, and smart accident assistance. Some uses of IoT-based traffic management include Smart parking, as getting area for parking in urban areas are time consuming. The smart parking system could be modeled using IoT hardware, RaspberryPi and Arduino boards. Real-time information regarding void parking space for vehicle can be acquired by the IoT rooted sensors. Traffic lights are another application in which Sensors that are positioned strategically are able to apply technology of IoT to extract data. The data collected can be examined to give drivers with alternative paths and traffic signals will be improved. Road-side lights is the application of IoT based traffic control system in which Smart lights could be applied by employing environmental sensors for increasing or reducing brightness suitable to present light situations. Smart assistance is also an application in which accidents and extremities can be recognized using sensors and CCTV in the lanes and send their locality to teams of emergency.

\section{CONCLUSION}

Nowadays, Traffic congestion is the important issue in the case of transport system in the countries which are still developing. This allows rise in fuel expenditure and also leads to air contamination. The combination of technology of computer vision with that of IoT aids in building intelligent system that can control traffic in an efficient way. A few effective traffic system networks are built which gives drivers with real-time update about the state of roads through a website. IoT rooted smart roads improve commuting time, safety on road and decrease traffic jams. These systems can be executed using any complex algorithms. These system set up costs less and they are movable systems which leads to reduced traffic crowding. The mechanized system decreases time spent by travellers on the traffic signals and also decreases traffic crowding on busy roads. It also guarantees safety of pedestrians.

\section{FUTURE WORK}

The system has to be specially made for remote observing and management of traffic on roads. some works of researchers needs to be improved by building website for observing and managing separately by node -red dashboard. The systems can support many combinations like detection of event and failure alert message. Different systems like parking machines, traffic counting stations and city observing camera when integrated provides a good management. Real-time categorizing of information on various kinds of vehicles can be made possible by allowing the whole vehicle testing procedure. Experiments needs to be expanded to private cloud in the direction of areal fog computing. With the aid of the augmented reality important information like speed and path of movements can be observed. This would give on to a city with smart and high-tech framework. The traffic control system using IoT is an important step which can lead to the building of smart cities in the future.

\section{REFERENCES}

1. Mounir BOUHEDDA, Hamza BENYEZZA, Ibrahim METALI, Sofiane TCHOKETCH KEBIR, Samia REBOUH, "Traffic Intersection Fuzzy Control Supervision System based on IoT", The $3^{\text {rd }}$ International Conference on Applied Automation and Industrial Diagnosis(ICAAID), 2019.

2. Anilloy Frank, Yseer Slim Khamis Al Aamri, Amer Zayegh, "IoT based Smart Traffic density Control uding Image Processing”, IEEE, 2019.

3. Satbir Singh, Baldev Singh, Ramandeep, Baljit Singh, Amitava Das, "Automatic Vehicle Counting for IoT based Smart Traffic Management System for Indian urban Settings", IEEE, 2019.

4. Mehal Zaman Talukder, Sheikh Shadab Towqir, Arifur Remon, Hasan U. Zaman, "An IoT Based Automated Traffic Control System With Real-Time Update Capability", $8^{\text {th }}$ ICCCNT July 3-5 IIT Delhi, India, IEEE, 2017.

5. Pampa Sadhukhan, Firoz Gazi, "An IoT based Intelligent Traffic Congestion Control System for Road Crossings", International Conference on Communication, Computing and Internet of Things(IC3IoT), IEEE, 2018.

6. Anna Merine George, V.I.George, Mary Ann George, 'IoT based Smart Traffic Light Control System", International Conference on control , Power, Communication and Computing Technologies(ICCPCCT), IEEE, 2018.

7. Varsha Sahadev Nagmode, Prof.Dr.S.M.Rajbhoj, "An IoT Platform for Vehicle Traffic Monitoring System and Controlling System based on Priority", $3^{\text {rd }}$ International Conference on Computing, Communication, Control and Automation(ICCUBEA), IEEE, 2017.

8. Rafik Zitouni, Jeremy Petit, Aghiles Djoudi, Lauren George, "IoT based Urban Traffic Light Control: Modelling, Prototyping and Evaluation of MQTT protocol", International conference on Internet of Things and IEEE green computing and IEEE smart data, 2019. 
9. Saifuzzaman, M., Khan, A.H., Moon, N.N., Nur, F.N. (2017). "Smart Security for an Organization based on IoT", International Journal of Computer Applications 165(10), 33-38.

10.Zu, L.D., He, W., \& Li, S. (2014)," Internet of Things in Industries: A Survey" IEEE Transactions on Industrial Informatics, 10(4).doi: 10.1109/TII.2014.2300753

11. Cho, S. K. and Dhingra, V. (2008). "Street Lighting Control Based on Lon Works Power Line Communication," IEEE International Symposium on Power Line Communications and Its Applications, Jeju City, 2-4 April 2008, pp. 396- 398.

12. S. Kumar Janahan, M. R.M. Veeramanickam, S. Arun, K. Narayanan, R. Anandan and S. Javed Parvez, "IoT based smart traffic signal monitoring system using vehicles counts", International Journal of Engineering and Technology, vol. 7, no. 221, pp. 309, 2018.

13. P.V Ashok, S SivaSankari and V. M. S. Sankaranarayanan, "IoT Based Traffic Signalling System", International Journal of Applied Engineering Research, vol. 12, no. 19, pp. 8264-8269, 2017, ISSN 0973-4562

14. N. Choosri, Y. Park, S. Grudpan, P. Chuarjedton and A. Ongvisesphaiboon, "IoT-RFID Testbed for Supporting Traffic Light Control", International Journal of Information and Electronics Engineering, vol. 5, no. 2, March 2015.

15. M. Lakshminarasimhan, "Advanced Traffic Management System Using Internet of Things", Researchgate, March 2016. 